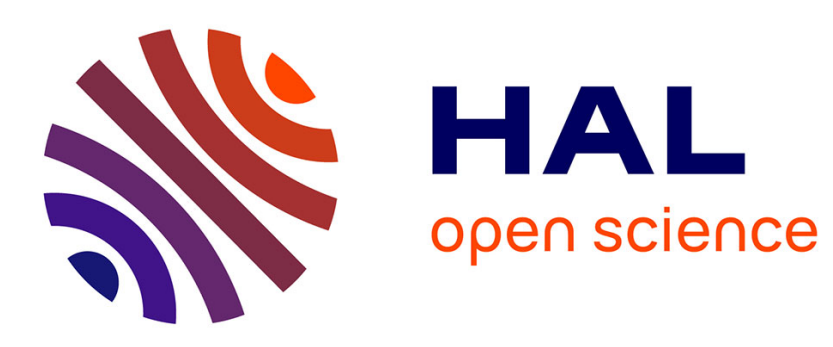

\title{
Progress on Self-mixing Sensors for In-situ Displacement Measurement
}

\author{
Antonio Luna Arriaga, Thierry Bosch, Francis Bony
}

\section{To cite this version:}

Antonio Luna Arriaga, Thierry Bosch, Francis Bony. Progress on Self-mixing Sensors for In-situ Displacement Measurement. Electronics, Control, Measurement, Signals and their application to Mechatronics (ECMSM), 2013 IEEE 11th International Workshop of, Jun 2013, Toulouse, France. pp.1-5, 10.1109/ECMSM.2013.6648959 . hal-00937950

\section{HAL Id: hal-00937950 https://hal.science/hal-00937950}

Submitted on 13 Feb 2014

HAL is a multi-disciplinary open access archive for the deposit and dissemination of scientific research documents, whether they are published or not. The documents may come from teaching and research institutions in France or abroad, or from public or private research centers.
L'archive ouverte pluridisciplinaire HAL, est destinée au dépôt et à la diffusion de documents scientifiques de niveau recherche, publiés ou non, émanant des établissements d'enseignement et de recherche français ou étrangers, des laboratoires publics ou privés. 


\title{
Progress on Self-mixing Sensors for In-situ Displacement Measurement
}

\author{
Antonio Luna Arriaga ${ }^{1,2}$, Francis Bony ${ }^{1,2}$ and Thierry Bosch ${ }^{1,2}$ \\ ${ }^{1}$ CNRS, LAAS, 7 avenue du colonel Roche, F-31400 Toulouse, France \\ ${ }^{2}$ Univ de Toulouse, INP, LAAS, F-31400 Toulouse, France \\ alunaarr@laas.fr
}

\begin{abstract}
This document presents a survey on reported devices exploiting the emerging technology of self-mixing interferometry for non-contact displacement reconstruction. Nowadays, there is a broad diversity of signal processing methods over self-mixing devices, making difficult to decide which one would satisfy a given application domain. It has been observed that a change in the requirements specification implies a complete redesign of the system. Therefore, this analysis aims to identify the characteristics and features of actual systems in order to narrow the path to take towards the development of an embedded sensor. For this study, we consider the systems promising a robust usage for operation in a non-conditioned environment. We present the tradeoffs between complex signal processing, the usage of external components and precision of the device. From this report we observe that maintainability and scalability still penalize overall system deployment and we suggest that should be considered for time-to-market solutions.
\end{abstract}

Keywords-Displacement measurement; optical feedback; Instrumentation and measurement

\section{INTRODUCTION}

Non-contact measurement of moving targets by using selfmixing (SM) interferometry with laser diodes (LD) is a promising low-cost instrumentation scheme. The main observable research areas are: absolute distance, displacement, velocity and vibration measurement [1]. In mechatronics field, some demonstrations are: modal analysis [2], and structural damping evaluation [3].

The basic configuration (Fig.1) consist on a LD as the source, a photodiode (PD) as the optical output power monitor and a collimating lens to focalize the projected beam on the surface under measurement. In this self aligned setup, a small amount of light is back-scattered into the active laser cavity inducing a modulation in frequency and amplitude of the emitted beam [4]. By exploiting this self-mixed signal it is possible to retrieve information for the above mentioned physical parameters. Naturally, each research domain performs state-of-the-art techniques to improve their measurements or to explore new opportunity areas for this instrumentation. As expected, there is a wide variety of proposals on literature, however just a few of them are targeted to be conceived as an end user device (i.e. robust and easy operation conditions). Furthermore, the systems concerned are fine tuned for a given application, in most of the cases a change in the requirements specification implies their complete redesign [5].

This report concerns the devices performing a displacement reconstruction by SM. In such a setup, the most considerable requirements modifications can be: the distance between LD

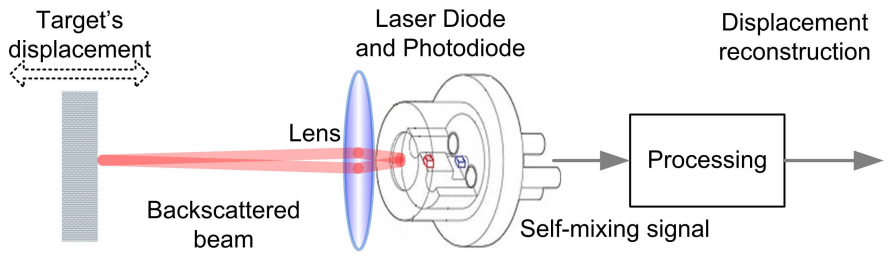

Fig. 1. The basic self-mixing setup: a laser diode with a built-in photodiode, a collimating lens and a means to exploit the monitored interferometric signals.

and target, the amplitude of displacement, the system's bandwidth and the coupling with the surface's reflectivity. For a narrow analysis, we consider the published devices the last 10 years promising a robust usage, so a non skilled in the art person can be able to perform a measurement. The aim is to observe the tradeoffs on system complexity considering overall development effort. It attempts to identify the trends on this field to allow this technology to be approached as an embedded displacement sensor.

\section{DISPLACEMENT INFORMATION RETRIEVAL}

The interaction between the emitted laser beam and the optical retro-reflections is the working principle of SM interferometry [1]. One parameter of special interest in the equations describing this phenomenon is the feedback coupling factor (C). This adimensional criterion, helps to describe the strength of the coupling field and has been largely studied to classify the feedback effects in semiconductor laser under retro-reflections. A weak level corresponds to a sine-like waveform of the monitored optical phase shift and is found for values between $0.1<C<1$. The moderate regime presents a sawtooth-like waveform for the range of $1<C<4$.6. After this upper limit the laser is considered as being under strong feedback. This last regime is much more difficult to exploit since there are chances that some interferometric fringes get vanished on the low frequency carrier of the SM signal. For $C>1$, the hysteresis phenomenon rises. This affects the average energy of the interferometric fringes, leading to complex signal processing as the presented in [6]. It needs to be mentioned that SM phenomenon is extremely sensitive and the $\mathrm{C}$ value can slightly change for a given application. Therefore, a change on the shape in time and hysteresis of the signal can be expected.

The information about the amount of displacement amplitude, as well as the direction when the target is approaching or moving away along the laser beam axis, can be understood from Fig.2. It depicts simulated SM signals for the same kind of displacement but at different $\mathrm{C}$. The method used to create 

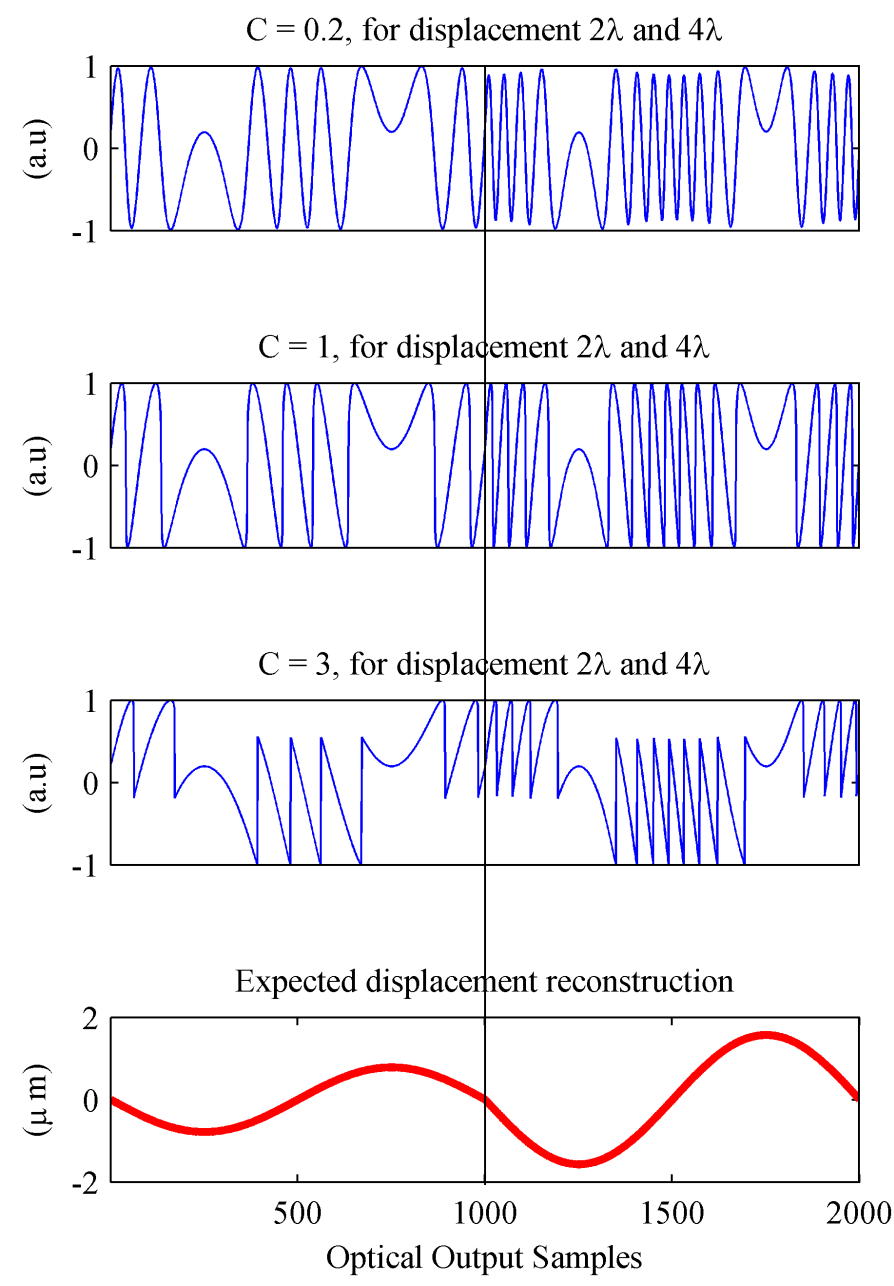

Fig. 2. Simulated self-mixing signals at different feedback levels and the target's displacement performing a sinusoidal movement increasing in amplitude.

the simulated SM signals is explained in Fig. 2 from [3]. On the bottom, it is shown the target's displacement originating the interferometric fringes in number of times the LD's wavelength ( $2 \lambda$ and $4 \lambda$ for this example). As outlined by the left and right sinusoidal movements, the number of interferometric fringes increased from 4 to 8 , as the the amount of peak-to-peak amplitude increased. From the same figure for $C=3$ it is easier to identify the target's movement direction with the upward and downward ramp of the saw-tooth like signals.

Displacement reconstruction relies on two main blocks: a) the identification of each interferometric fringe at the proper phase and direction, $b$ ) the precision reached on the analysis of nanometric excess $(\varepsilon)$ of a fringe. As in conventional interferometry, the fringe period corresponds to a target displacement of half wavelength of the LD (e.g. $\lambda_{o} 400 \mathrm{~nm}$ in the near infrared). To quantify the motion in one direction it is possible to use $D=N\left(\frac{\lambda_{o}}{2}\right)+\varepsilon$, where $\mathrm{N}$ is the number of fringes observed and $\lambda_{o}$ the wavelength of the laser beam without feedback. A coarse measurement in $\lambda_{o} / 2$ precision can be achieved by a system detecting the fringes and integrating them in order to reconstruct the displacement [1]. The increase of resolution implies a major understanding on the physics behind SM phenomenon in order to propose more complex techniques.

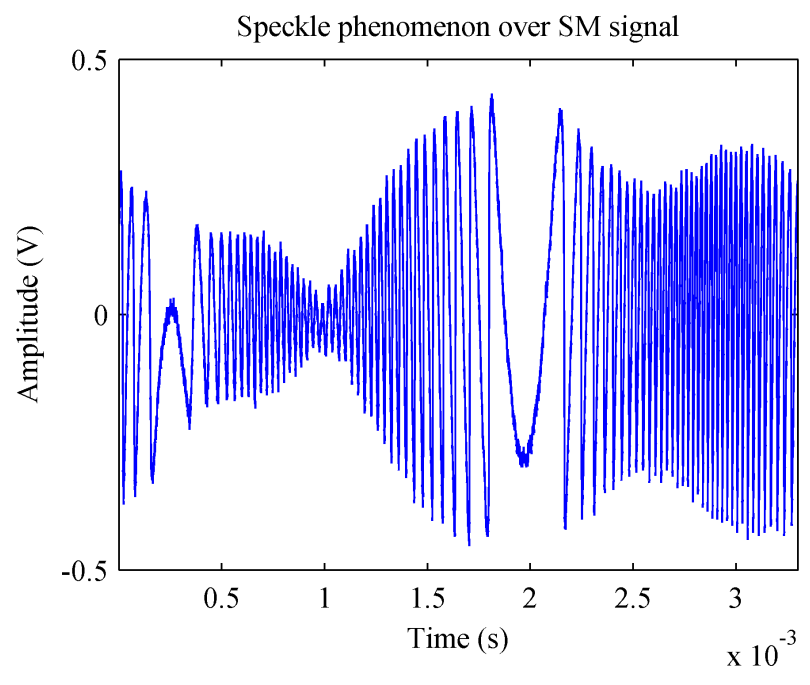

Fig. 3. The random amplitude attenuation by speckle phenomenon.

The evaluation of precision methods is out of the scope of this work, which considers only those methods validated on a materialized system. It is worth to mention that analog signal reconstruction in the range of 1-100 $\mu \mathrm{m}$ with sub-wavelength precision is advised for easy elaborations [5].

Another undesired effect over self-mixing displacement signals is the attenuation by speckle-pattern statistics. This phenomenon occurs with the random phase superposition of the back-scattered waveforms when the illuminated surface presents roughness relative to the fixed spot size [4]. In practice this is observed as random dark and bright spots where the laser beam falls. In Fig.3, we acquired a real SM signal under experimental conditions to illustrate the phenomenon. The target is a speaker cone coated with a slow-fading material to increase the probability of phase superposition. It is driven to perform a sinusoidal movement with a period of $3.3 \mathrm{~ms}$ for a displacement of nearly $30 \mu \mathrm{m}$ on the full swing. From this figure, it can be seen the envelope over the amplitude induced by speckle phenomenon. Within this condition the measurement can be unreliable as a result of possible fringe count loss by a threshold based technique. In severe speckle conditions, signal amplitude can decrease up to fading at certain points, therefore robustness of a system is correlated to its capacity to operate at different surface conditions.

The following section presents, to our knowledge, the published devices meeting the criteria for in-situ measurement. The information is presented in chronological order and entitled by a distinguishable characteristic of the sensor, in the case of evolutive papers, the latest reported device is listed.

\section{AnALYZED SElF-MiXing SENSORS}

\section{A. Speckle Tracking (ST)}

By using piezoceramic actuators, this system tracks the brighter spots in the $\mathrm{x}-\mathrm{y}$ plane to ensure a maximum amplitude of the measured signal. A phase-sensing loop and a liquid crystal attenuator help to control the feedback level to a suitable exploiting condition (Fig.4). To exploit the signal processing, the system uses an analog derivative filter as front-end to extract the interferometric fringes. Then two solutions can be 


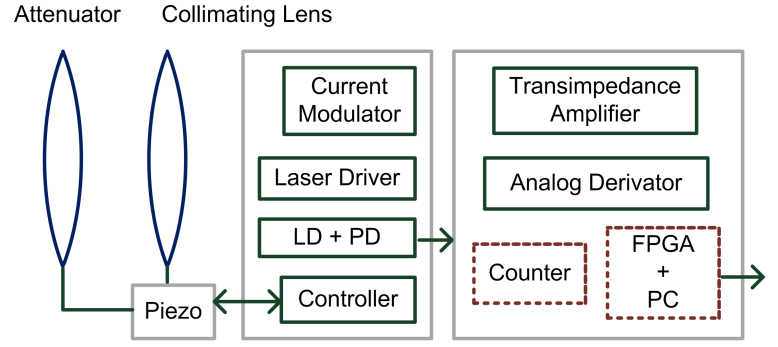

Fig. 4. Block diagram of the speckle tracking system.

used for the displacement measurement process. The analog version uses count-down buffers to measure the amplitude, while the digital version uses a field programable gate array (FPGA) and a computer (PC) for rendering information. The signal processing carried by the FPGA is based on an adaptive threshold and a fringe counter integrator. The program on the $\mathrm{PC}$ receives the counter information, multiplies by the corresponding $\lambda / 2$ factor and graphically renders the displacement information [7]

1) Precision: About $400 \mathrm{~nm}$.

2) Displacement range: Up to $500000 \mu \mathrm{m}$.

3) Movement frequency: From $100 \mathrm{~Hz}$ to $1 \mathrm{KHz}$.

4) Robustness against speckle: Attained by the transverse micro-displacements of the laser beam.

5) Validation environment: Experimental in real-time, with ease of use.

6) Feedback level: The used technique avoids obtaining weak feedback from several materials. The signal processing is well suited to treat moderate feedback.

\section{B. Fringe Locking (FL)}

This system uses an electronic feedback loop acting on the LD wavelength in order to keep constant the phase of the signal. In order to track the interferometric fringes, the operational amplifier (OP-AMP) is fed by the SM signal and the voltage level of half a fringe. The output of this amplifier in differential mode is converted to current and used to feed the LD (Fig.5). In order to calculate the proper magnitude, the distance between LD and target is measured as pre-requisite by the same system. To this end a range finding technique is used, it consists on driving the LD by a triangular current modulation over one cycle and calculate the displacement of the generated fringes. By means of a low-pass filter and the feedback loop, the target's displacement is reconstructed [8].

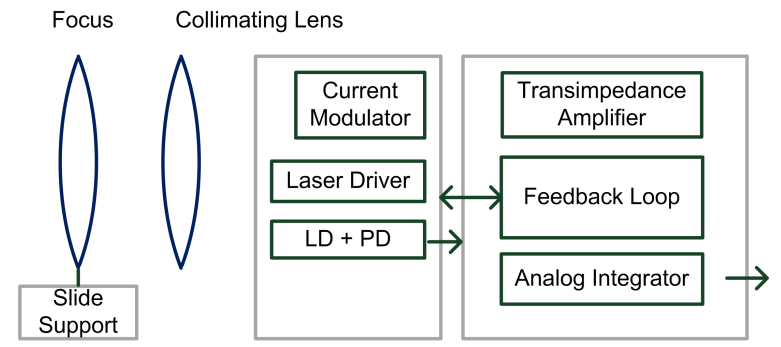

Fig. 5. Block diagram of the fringe locking system.
1) Precision: $0.1 \%$ of actual output signal reading (e.g. $180 \mathrm{~nm}$ for max. displacement).

2) Displacement range: Up to $180 \mu \mathrm{m}$.

3) Movement frequency: From $0.1 \mathrm{~Hz}$ to $70 \mathrm{KHz}$.

4) Robustness against speckle: The optical (cumbersome) arrangement in this device helps to attain an efficient coupling for a variety of surfaces.

5) Validation environment: In-situ, the laser spot must be adjusted to obtain a convenient signal amplitude before measuring.

6) Feedback level: The preferred working condition is the moderate feedback.

\section{Switching Algorithms (SA)}

This system uses a mechanical pickup similar to the one included on CD players, to move the collimating lens along its longitudinal axis at power-on or reset (Fig.6). It uses current modulation on the LD as the previously described fringe locking system. By means of this autofocus process, the maximum amplitude of the SM signal can be reached. After locking the best lens position, a peak finder algorithm decides between applying an algorithm suited for weak feedback or another algorithm for moderate feedback. The algorithm for $C<1$ calculates the duty cycle (DC) of the interferometric fringe to affect its contribution on the reconstructed displacement towards or away the LD. The second algorithm performs an unwrap of the SM signal at the trigger points obtained by the peak finder algorithm. Finally the displacement is retrieved in analog form [6].

1) Precision: About $400 \mathrm{~nm}$ for weak feedback and $50 \mathrm{~nm}$ for moderate feedback.

2) Displacement range: Up to $10000 \mu \mathrm{m}$, limited by the focus depth.

3) Movement frequency: Inferred $7 \mathrm{~Hz}$ max. for a sampling rate of $1 \mathrm{MHz}$.

4) Robustness against speckle: Performed by the autofocus system before a measurement and by DC algorithm for weak feedback.

5) Validation environment: Experimental in real-time, with apparent ease of use.

6) Feedback level: The signal processing is well suited to treat weak and moderate feedback.

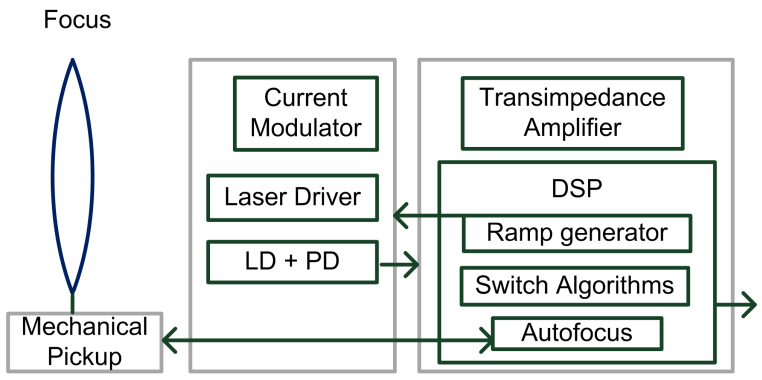

Fig. 6. Block diagram of the switching algorithms system. 


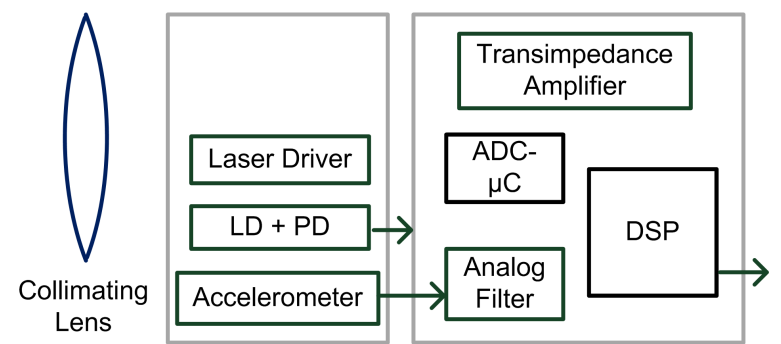

Fig. 7. Block diagram of the accelerometer assisted system.

\section{Accelerometer Assisted (AA)}

This system uses a solid state accelerometer (SSA) disposed next to the LD in order to substract extraneous movements affecting the displacement reconstruction of the remote target. This particular setup opens the possibility for embedding this technology to non-stationary support (e.g. optical table or anti-vibration material). The SM signal is treated by a micro-converter device delivering a displacement signal containing the parasitic movements. On the other hand, the accelerometer displacement is retrieved by a double integration performed into standard operational amplifiers. Both displacement outputs are fed into a digital signal processor (DSP) for proper substraction Fig.7. The DSP performs phase and gain adjustments to produce the final target displacement [9].

1) Precision: About $96 \mathrm{~nm}$, dependant on SSA noise characteristics.

2) Displacement range: Tested for $20 \mu \mathrm{m}$.

3) Movement frequency: From $40 \mathrm{~Hz}$ to $500 \mathrm{~Hz}$.

4) Robustness against speckle: Not directly reported.

5) Validation environment: Experimental in real-time, with ease of use.

6) Feedback level: From the reported method, it is inferred that SM signal processing is best suited to moderate feedback.

\section{CRitical Analysis}

The presented devices respond to the requirements for a robust usage, however the diversity of design approaches requires to establish further criteria in order to support the decision of producing a sensor for end users. The following subsections deal with the observed system tradeoffs, is the authors' suggestion to consider them on the requirements specification of self-mixing sensors with the interest of preserving it as low-cost and easily deployable technology.

\section{A. External Components}

Perhaps the most noticeable characteristic from the above devices is the usage of external components to the basic SM configuration. While this brings stability on the measured signal, it is important to consider the impact on the bulkiness and price on the manufacturing process. Usually optical parts are more expensive face to electronic components, in fact that is one of the reasons of the popularity of SM interferometry face to the traditional interferometers. The FL system, presents a fair agreement in terms of development price, in part by its simple optical arrangement. However the distance separating the collimating lens from the focusing lens prohibits its usage on size-restricted devices. The mechanical solution in ST, suggests a controlled environment on the sensor head to allow the micro-displacement tracking to work properly. Therefore an indirect cost of deployment is to be considered. The devices SA and AA seem to better satisfy the constraints of price and size, being privileged for embedded applications. It is observed that devices ST and FL deal with the signal stability by using external components, allowing a simpler signal processing. On the other hand, devices SA and AA increase the complexity of the treatments at expenses of reducing the bulkiness of the components.

\section{B. Signal Processing}

As stated in section II, displacement reconstruction in coarse resolution is the easiest signal processing to implement. While more precision is required a variety of solutions rise and the choice does not seem evident. An aspect that might assist this task, is to consider the maintainability of the designs. The ST system uses a simple method for its analog implementation and similar approach for the digital version thanks to the good signal quality obtained by external components. The FL system presents an interesting analog electronic design. Thought simple in principle, it requires a careful design of each of the steps and the knowledge of the underlying equations for the displacement calculation. The devices SA and AA use more complex algorithms, however they are implemented mostly on digital processors allowing more flexibility and reproductivity of the devices.

\section{Precision and Bandwidth}

As mentioned in the introduction, a modification of the target's movement condition may affect the precision as observed on SA system. Even if devices ST and FL are robust face to speckle phenomenon, they are less precise than the elaborations SA and AA using more complex signal processing. The fully analog system in FL is the one offering more bandwidth, thus suitable for more applications. The good maintainability of digital implementations is then compromised by the sampling frequency of analog-to-digital converters and the proper handling of the acquired signal. However, the implementations in SA and AA offer more flexibility to be scaled for measuring bigger amplitude displacements. When precision and bandwidth are not strictly defined a digital approach shall be privileged.

\section{Mechanical Displacement Measurements}

For the sake of illustration, let us show two displacement reconstructions utilizing the real-time algorithm from [9]. The moving target is a Piezo-electric Transducer (PZT) from Physik Instrumente, comporting a built-in capacitive feedback reference sensor and a resolution of $2 \mathrm{~nm}$. On Fig.8 the PZT is driven by a sinusoidal signal at $70 \mathrm{~Hz}$ and $2 \mathrm{~V}$ peak to peak (magenta signal on the middle), providing a displacement of $5 \mu \mathrm{m}$ of amplitude. On the bottom it can be observed the corresponding twelve interferometric fringes of SM signal for each amplitude swing. The top signal depicts the reconstructed target displacement from the SM signal, thus, demonstrating 
TABLE I. COMPARISON OF ANALYZED SYSTEMS IN TERMS OF DESIGN TRADEOFFS.

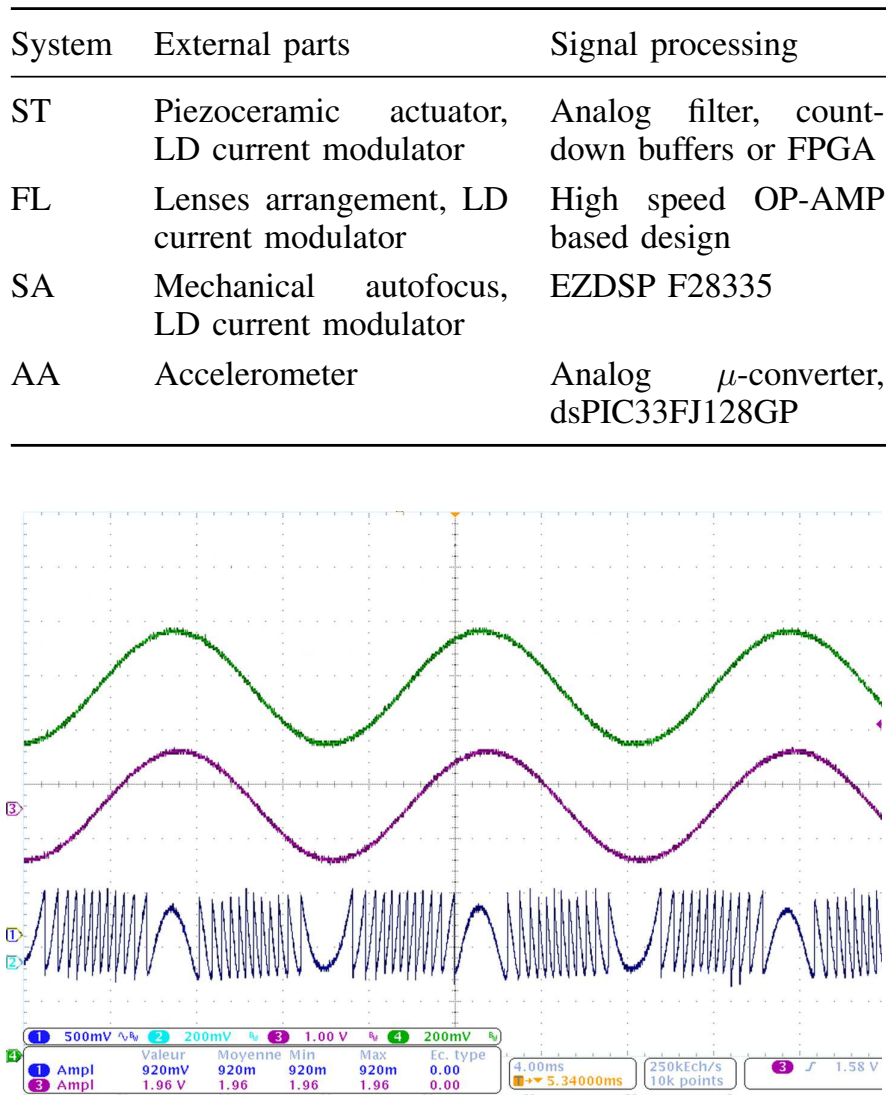

Fig. 8. Sinus displacement reconstruction with SM sensor (top) and with reference sensor (middle), corresponding SM signal (bottom).

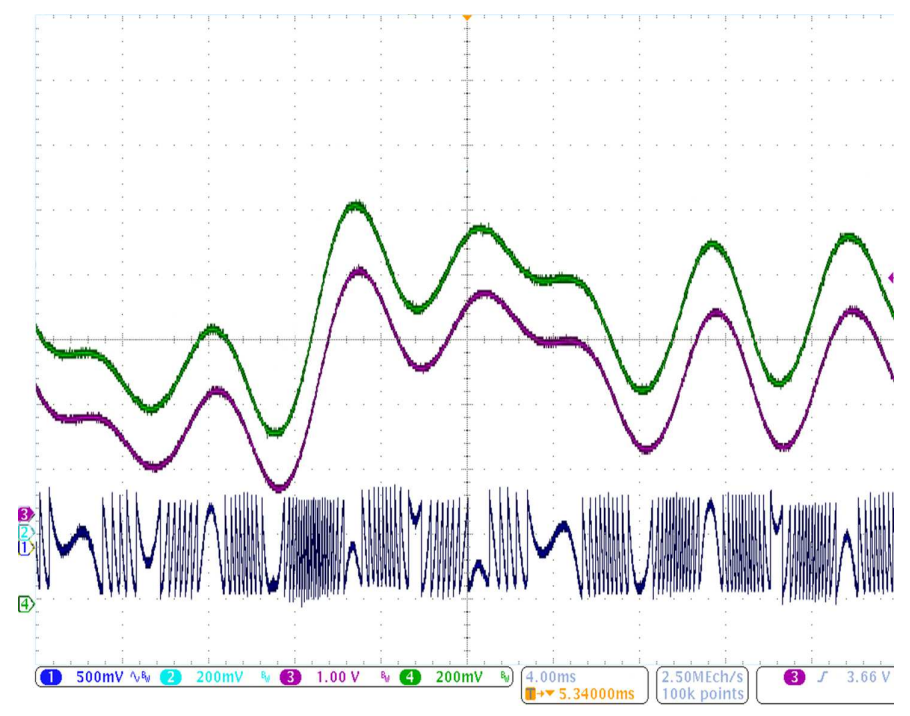

Fig. 9. Aleatory displacement reconstruction with SM sensor (top) and with reference sensor (middle), corresponding SM signal (bottom).

the interest of this instrumentation scheme. Fig.9 shows instead, an arbitrary movement driving the PZT composed of sinusoidal signals at different frequencies and amplitudes. It can be observed on the bottom SM signal how the number of fringes increases with the amplitude of displacements as previously shown on the simulation of Fig.2. The interest
Main observed feature

Validation movement

The biggest amplitude Sinus $+\quad$ manual

measurement

displacement on a slit

The best bandwidth

Sinus, triangular, squared from speaker

Performance for weak and Sinus from speaker moderate feedback

The less bulky and nonconstrained to stationary support

Sinus + arbitrary mounted on a shaker

of validating the systems also for arbitrary displacements is to observe the robustness of the design as it could be used for broaden applications, rather than just for vibration-related instrumentation.

\section{SUMMARY AND CONCLUSION}

Table I summarizes the analysis carried on previous section for the listed devices. In order to decide a design strategy for a given application, we have proposed the following criteria (other than precision and bandwidth) to assist the choice: 1) bulkiness of the device, 2) operation environment (e.g. industrial or controlled), 3) scalability and maintainability of the design. We enlighten that overall deployment of the technology shall be taken into account in order to preserve the premise of SM being a low-cost sensing scheme. The trend foreseen for SM sensors is in the flexibility to adapt the systems requirements to provide time-to-market solutions.

\section{REFERENCES}

[1] T. Bosch, C. Bes, L. Scalise, and G. Plantier, "Optical feedback interferometry," in Encyclopedia of Sensors. American Scientific Publishers, 2006, vol. 7, pp. 107 - 126.

[2] N. Servagent, T. Bosch, and M. Lescure, "A laser displacement sensor using the self-mixing effect fot modal analysis and defect detecion," IEEE Trans. Instrum. Meas., vol. 46, no. 4, pp. 847 - 850, aug. 1997.

[3] C. Bes, V. Belloeil, G. Plantier, Y. Gourinat, and T. Bosch, "A selfmixing laser sensor design with an extended kalman filter for optimal online structural analysis and damping evaluation," IEEE/ASME Trans. Mechatronics, vol. 12, no. 3, pp. 387 - 394, jun. 2007.

[4] G. Giuliani, M. Norgia, S. Donati, and T. Bosch, "Laser diode selfmixing technique for sensing applications," Journal of Optics A: Pure and Applied Optics, vol. 4, pp. S283 - S294, 2002.

[5] S. Donati, "Developing self-mixing interferometry for instrumentation and measurements," Laser Photonics, vol. 6, no. 3, pp. 393 - 417, 2012.

[6] A. Magnani, A. Pesatori, and M. Norgia, "Self-mixing vibrometer with real-time digital signal elaboration," Applied Optics, vol. 51, no. 21, pp. 5318 - 5325, 2012.

[7] M. Norgia, S. Donati, and D. D'Alessandro, "A displacement-measuring instrument utilizing self-mixing interferometry," IEEE Trans. Instrum. Meas., vol. 52, no. 6, pp. 1765 - 1770, dec. 2003.

[8] G. Giuliani, S. Bozzi-Pietra, and S. Donati, "Self-mixing laser diode vibrometer," Measurement Science and Technology, vol. 14, pp. 24 - 32, 2003.

[9] U. Zabit, O. D. Bernal, A. Chamorro-Coloma, and T. Bosch, "Realtime accelerometer coupled self-mixing laser displacement sensor for embedded applications," in IEEE Sensors Conference, 2012, pp. 1 - 4. 